\title{
Application Tabu Search in Matlab for Optimize Distribution Systems
}

\author{
J. Domínguez Navarro* IEEE Member, A. Bayod Rújula** IEEE Member, \\ O. Coronado De Koster ${ }^{* *}$ IEEE Student Member \\ ${ }^{*}$ U. de Zaragoza (Department of Electric Engineering) ${ }^{* *}$ U. Autónoma de Tamaulipas (Production Systems Engineering)
}

\begin{abstract}
Unlike the exact mathematical methods, the heuristic ones are different to provide a solution very near the optimal one, saving time of processor. With the appearance of the Tabu Search, by Fred Glover in 1986, diverse applications have arisen from the procedure to solve diverse problems as for the classic problem of the route of the vehicle (also known as the travelling agent problem) and the allocation of a plant. Here an adaptation to an existing code appears, applied to the problem of the travelling agent, for the reconfiguration of distributed generation units in three examples of distribution networks. The model provides results optimal or very near them in time of relatively little calculation, in comparison with the manual reconfiguration.
\end{abstract}

Index Terms-Tabu search, optimal allocation of distributed generation, minimization of power losses, approximate solution.

\section{ABBREVIATIONS}

TS $=$ Tabu Search

TSP $=$ Travel Salesman Problem

DGAP = Distributed generation allocation problem

$\mathrm{SD}=$ System Distribution

$\mathrm{GA}=$ Genetic Algorithm

DG $=$ Distributed Generation

$\mathrm{CT}=$ Centre of Transformation

$\mathrm{L}=\mathrm{Load}$

TPL $=$ Total Power Losses

PFA $=$ Power Flow Analysis

$P_{i}, Q_{i}=$ Active and reactive power of the node ith.

$P_{s g}, Q_{s g}=$ Active and reactive power of substations and generators units.

$n=$ Buses that conform the system of distribution.

$n g=$ amount of generators in the distribution system (including substations).

$\beta=$ Active and reactive power supply and demand balance equation.

$V=$ vector of voltages complex in the system distribution.

$\ell=$ Vector of transmission line flow.

$\delta=$ Vector of voltages angles.

loc_bus = load node in the distribution system.

cam_bus = node of the centre of trans-formation in the distribution system.

loc_subs = node of the substations in the distribution system.

loc_maq = node of the generators in the distribution system.

lista $=$ profile of voltages in the buses ordered of minor to greater.

bus_tipo $=$ variable of the configuration of the distribution system.

maq_ubica $=$ node of the generators in the distribution system. maq_con $=$ variable control of generators. car_ubica = load node in the distribution system.

ten_tabu = tabu list

\section{INTRODUCTION}

$\mathrm{T}$ HE provision of electrical energy not base of exclusive form in the delivery of the energy, but that also must of being this one of good quality. This can be obtained adding DG units (generation less than 10 $\mathrm{Mw}$ ) throughout the SD [10]. Nevertheless, the use of DG in a SD to improve the quality energy can carry a negative impact if isn't in an optimal position.

The interest that appears around the improvement in the power quality with DG units has provided heuristic that provides a solution very near to the optimal. In [1] is made an application of the heuristic one of GA for a multi-objective problem of optimization, where it is possible to be selected between some possibilities to improve: the losses of distribution or transmission, feeder power factor and the profiles of voltage, among others.

In [2] describes an implementation of GA for the reconfiguration of a SD for the TPL minimization. It's based on whole programming for the closing and opening of switches and to reshape the SD obtaining itself a configuration very approximated to the optimal one. Obtains a reduction of TPL in a $10 \%$.

The development of projects focused to heuristic isn't limited the use of genetic algorithms. In [3] formulates a power losses minimization problem, optimizing the capacity and the location of DG, cradle in TS, restricting the optimization to one DG by position. Nara K, et. al., it discusses on TS to find how much distribution power loss can be reduced if the DG is assigned optimally in the demand of a SD.

In [4], Mori H., et. al., affirms "TS is a transition algorithm that stores the found solutions to discover better solutions under different conditions of restriction”. Apply the heuristic in parallel for the location of capacitor throughout a radial distribution demonstrating its effectiveness on GA and GA in parallel in terms of exactitude and computational rapidity.

\section{MATHEMATICAL MODEL.}

In the present document the adaptation of a TS code will be approached [5][6] designed for TSP [5] for DGAP solution, where at least one is a DG. The logic of the TSP has similar with the DGAP. In the TSP the objective is to diminish the cost of the total trip by means of the combination of trips. And the DGAP objective is to minimize the TPL by means of the minimal injection possible of power in each node of the SD. The objective function and its restrictions [11]: 


$$
\begin{gathered}
\min \varsigma=f\left(P_{i}, Q_{i}, n\right) \\
\text { subjet to: } \\
\beta\left(P_{s g} Q_{s g}, n g\right)=0 \\
P_{s g}^{\min } \leq P_{s g} \leq P_{s g}^{\max } \\
Q_{s g}^{\min } \leq Q_{s g} \leq Q_{s g}^{\max } \\
V^{\min } \leq V\left(P_{i}, Q_{i}, n\right) \leq V^{\max } \\
\ell(V, \delta) \leq \ell^{\max } \\
n^{\min } \leq n \leq n^{\max } \\
n g^{\min } \leq n g \leq n g^{\max }
\end{gathered}
$$

\section{OPERATION OF ALGORITHM}

Next will present a flow chart appear on the procedure that was used like reference for the solution of the DGAP; for its later adaptation to the code of TS-TSP:

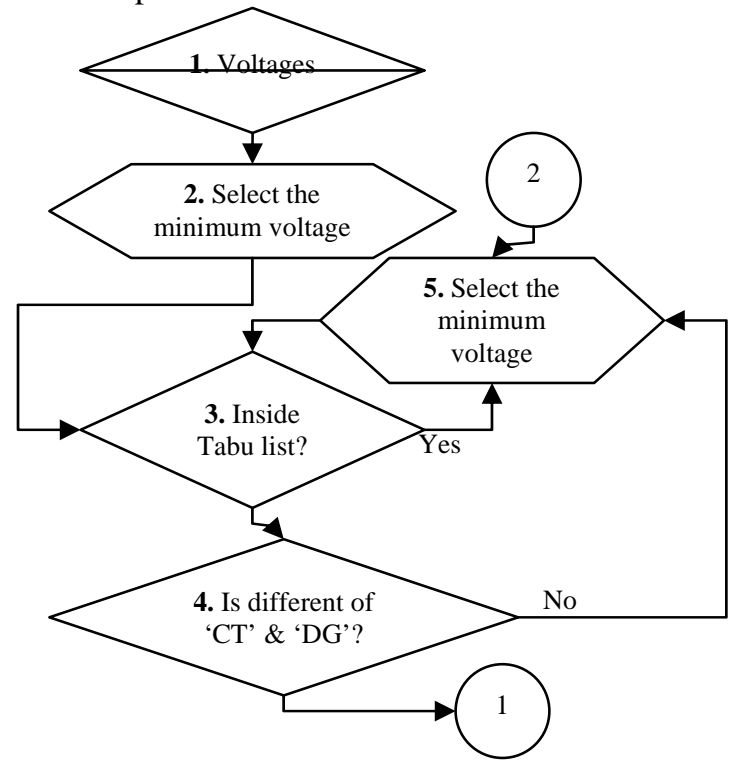

Fig. 1. Iterative process

The first step consists of the accomplishment of a PFA (fig 31) [8] with the initial configuration of the SD, to store the results of the configuration, location of $L$ and DG, in addition to the voltages in the buses and the TPL in the SD, like values objective. The voltages will be ordered ascending, lista, (fig 1-1) later to select the minimum voltage as reference and to initiate the changes in bus_tipo (fig 1-2), next will explain this process.

Once selected the minimum voltage, the position of the minimum voltage in the profile of voltages is stored that has left the power flow in loc_bus. While the stored data:

$>$ Isn't in the tabu list [9]. Fig 1-3.

$>$ Isn't in the node of a CT. Fig 1-4.

$>$ Isn't in the node of a DG. fig 1-4.

It's come closest to the search from the generator at the beginning of the SD and is stored in cam_bus (fig 2-6). In case that they are not fulfilled the rules previous become to the process from selection and the following member in list is taken, until the three mentioned rules are fulfilled (fig 1-5).

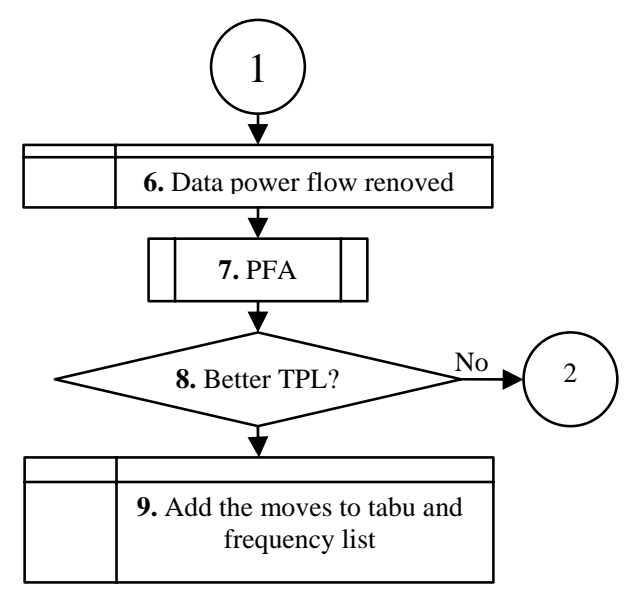

Fig. 2. Iterative process, continuation

Once established loc_bus and cam_bus, assigns like as DG and $\mathrm{L}$ in loc_bus and cam_bus, respectively, this in bus_tipo. The positions keep in two identifiers to later add them to the tabu list and to increase their frequency of repetition; to avoid cycles in the selection. Also, the values are modified in where now it's the generator in maq_ubica and maq_con, changing this value by loc_bus. Finally the values are modified in where now is the L, in the car_ubica variable, by the value of cam_bus. To this sequence it is denominated to him like movement. Fig 2-6.

In order to evaluate the performance of the conducted movement a PFA is executed updating: the type of bus, the location and way of control of the generators and the location of the loads in the SD, by means of the variables: bus_tipo, maq_ubica, maq_con, car_ubica, respectively (Fig. 2-7). It's evaluated if the TPL in the present movement are smaller to the TPL of the previous movement, of being therefore the objective values will be updated (Fig 2-8). Otherwise, the selection process is repeated taking into account the modified configuration and the profiles voltages. This process repeats so many times as the amount of elements that constitute the SD (fig 1-5). Finalized this process the movements conducted to tabu and frequency lists (fig 2-9). The frequency list consists of adding a unit whenever a movement is accepted and has its application in the diversification [6] (Fig. 3-5).

The next step in the optimization process is the aspiration criteria [5] (Fig. 3-4). It consists of analyzing if the current movement is within tabu list. If this movement is better than anyone of previously analyzed, the implied of the movement are eliminated of tabu tenure, and the objective values are updated (fig 3-9). Otherwise, the movement will be punished increasing its frequency of repetition and the value of the voltage in two units (user defined). When finding an optimal one in this point generally finalizes the iterative process. However, most of the times when arriving at this point are a local optimum, the exposed model excludes this instruction when finding an optimal (local or global) and continues making iterations in search of a better solution (Fig.3-4 section "yes"). 


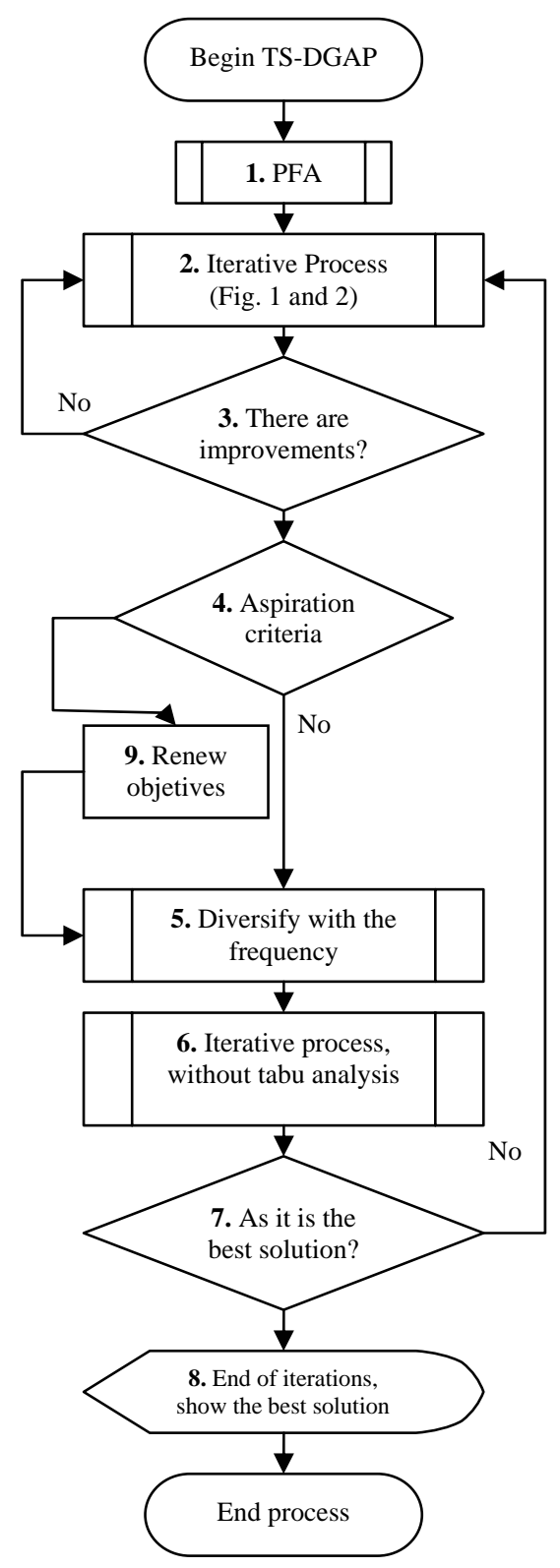

Fig. 3. Flow chart TS - DGAP

Like last passage of the TS procedure, the process of the diversification will be explained (fig 3-5). When it's reached a certain amount of iterations without improvement results, tabu list for the selection of the movements is ignored. Nevertheless, the movement will take place by means of the frequency matrix. Firstly taking loc_bus like the DG and cam_bus like the L with less frequency.

Executing a PFA again and comparing the TPL of the present movement with those of the previous movement and, if they are smaller, the objectives of the system are updated once again (fig 3-7). Of another form, it analyzes if the solution is the best one than it has been, and otherwise the model returns to the iterative process (fig 3-2). All process is repeated continuously until arriving at the end of the number of iterations that the programmer has designated (fig 3-8).

\section{RESULTS}

The first SD studied is a ring of distribution of medium voltage and account with one CT, three L, and two DG (fig 4); six buses.

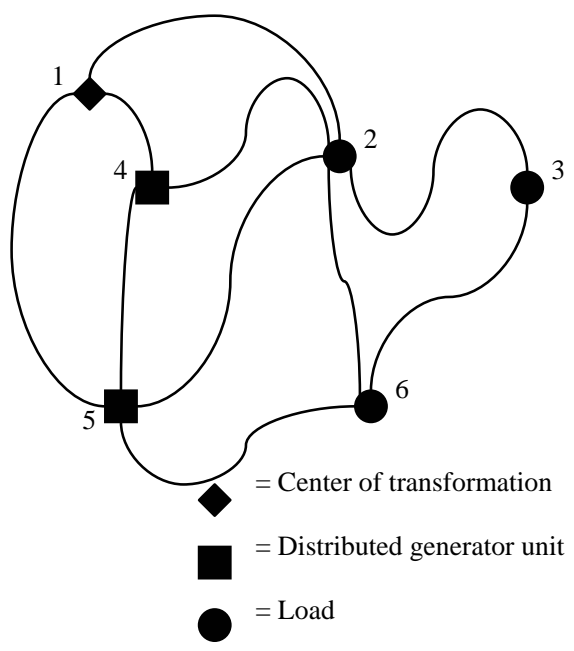

Fig. 4. SD Configuration

$\begin{array}{lcc}\text { Configuration } & \text { Voltages p.u. } & \text { TPL } \\ \text { Bus 1 - CT } & 1.0500 & \\ \text { Bus 2 - L } & 1.0000 & \\ \text { Bus 3 - L } & 1.0000 & \\ \text { Bus 4 - DG } & 1.0500 & 0.6861 \text { - j0.2762 } \\ \text { Bus 5 - DG } & 1.0700 & \\ \text { Bus 6 - L } & 1.0000 & \end{array}$

Table. I. Information of the SD initial configuration TS code provides information to the user of which it's happening in the iterative process, warning if there are or nonimprovements, the use of diversification, the number of iteration and the use of the aspiration criteria, among others. When finishing the optimization process shows the best obtained results (fig 5).

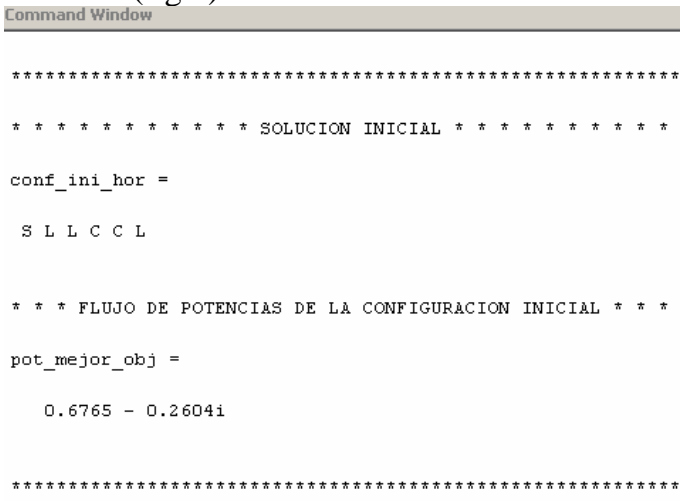




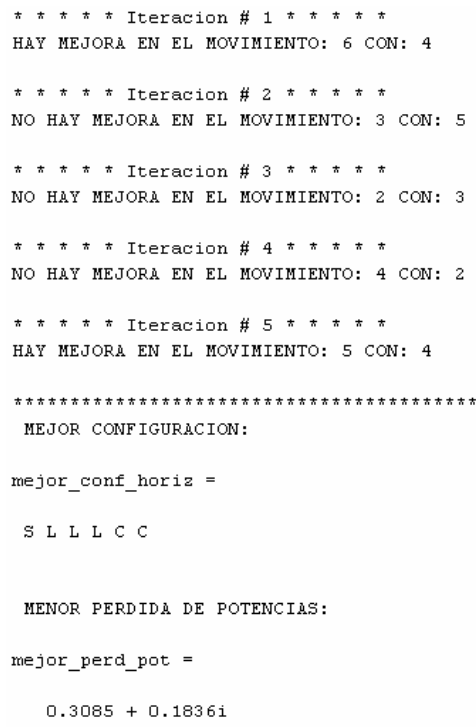

Fig. 5. Operation of code TS

The results appear of the same form in which the initial data were introduced, per unit (p.u.), the shown configuration in the end, is the smaller power loss obtained in SD. The other iterations just show if the movement had an improvement that satisfies the restrictions or isn't. If the aspiration criteria are used, like the diversification, is a text message.

Finally, two bidimensional graphs are generated that show the improvement through the iterations in TPL active (fig. 6a) and the reactive (fig. $6 b$ ).

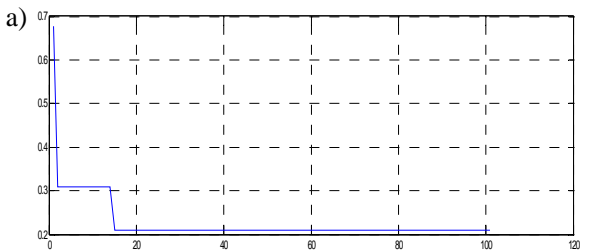

b)

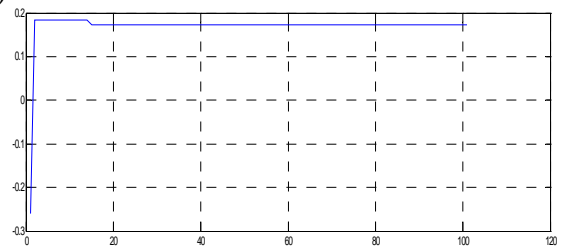

Fig. 6. Graph of results of TS-DGAP

a) Active power.

b) Reactive power

The efficiency of the model is explored using another SD like the previous, consisting of 13 buses with: $10 \mathrm{~L}$, two DG and one CT (fig 7).
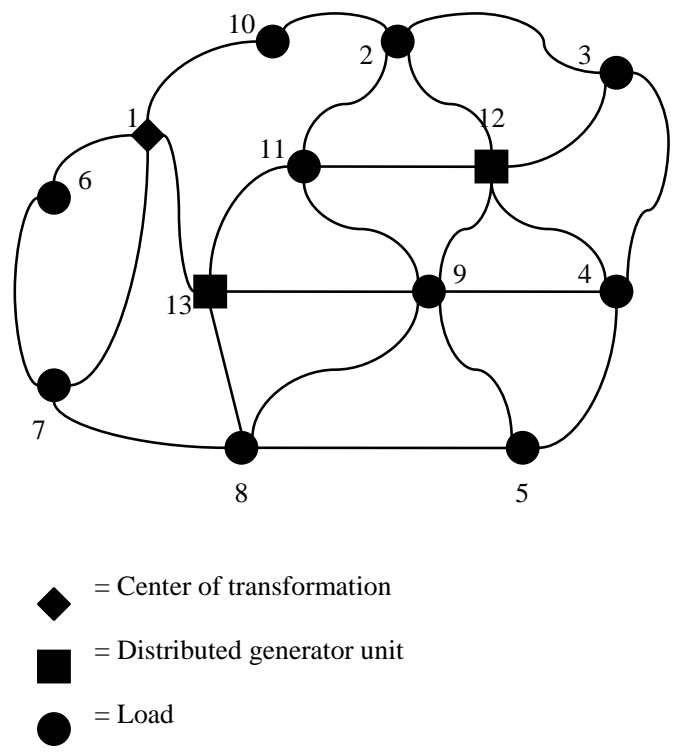

Fig. 7. TS - DGAP 13 buses SD.

Process TS-DGAP for this SD of 13 buses throws encouraging results in less than 20 iterations and can be seen in the next graph:
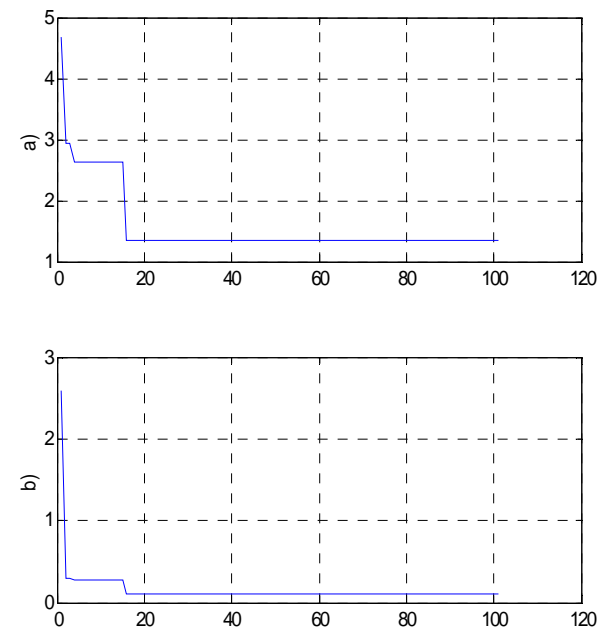

Fig. 8. After 100 iterations

a) Active power

b) Reactive power

Finally, TS-DGAP was proven with a radial SD of low voltage of 37 buses [12]. The SD shown in fig 9 is the model that is used generally to implement DG units: 


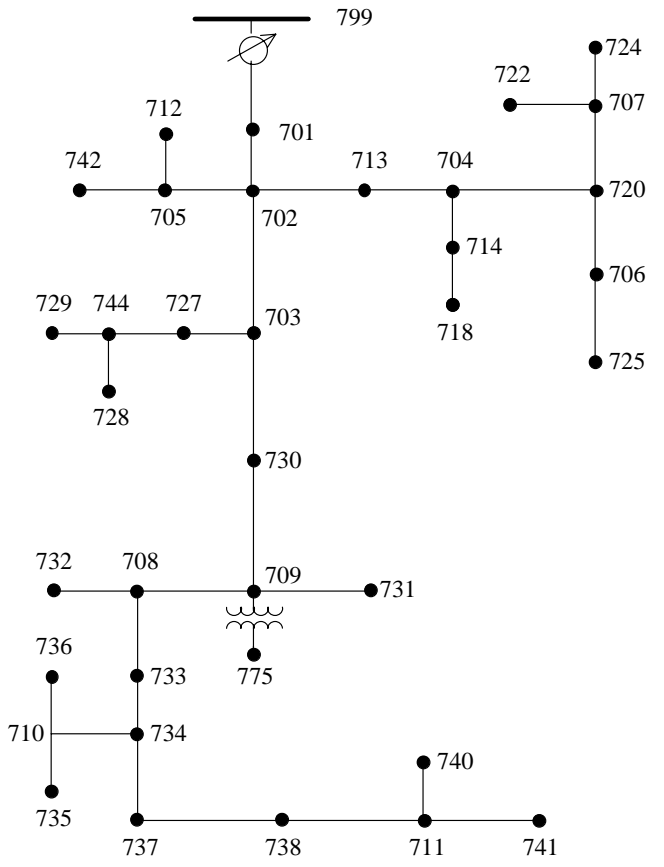

Fig. 9. IEEE 37 buses

Again, the program throws satisfactory results in a few iterations. Fig. 10.
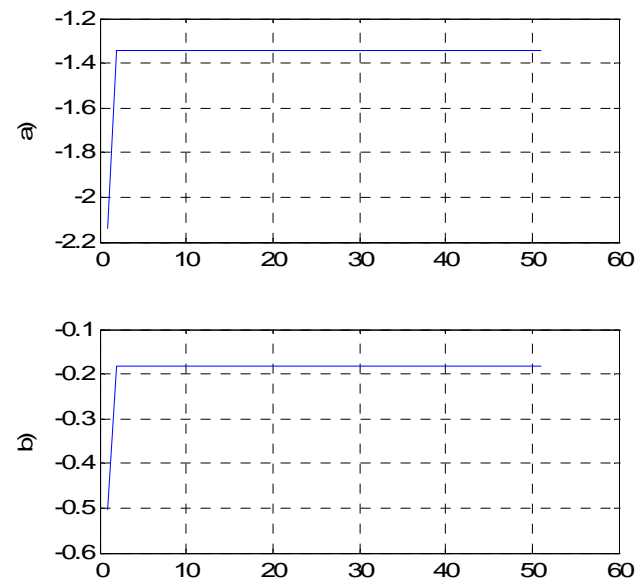

Fig. 10. TS - DGAP with IEEE 37 buses

a) Active power

b) Reactive power

But, if a DG is added to try to even diminish the TPL of the SD the problem increases of complexity. If a TS-DGAP is made adding a DG (0.15 p.u.) in position 720 , in addition to the DG ( 0.35 p.u.) located in 775 , the graph result like is next: Fit in emphasize that the movements always are made of selective form and using the configuration that has been used previously. Therefore the graphs of the results could seem not to be agreed to the results that a traditional TS provides. In the last figure it's even possible to be observed that sometimes there is an raise in some of the graphs, nevertheless in the other is a diminution, the TS-DGAP will try to balance both powers (it activates and it reactivates).

In agreement it increases the amount of buses that constitute the SD increases the time of processes used to make the optimization.
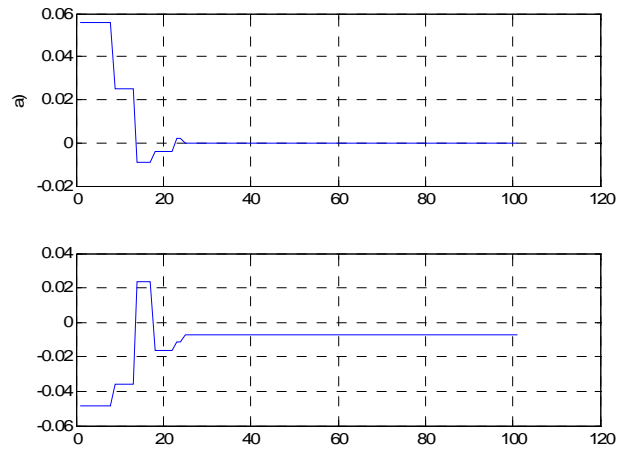

Fig. 11. Results of TS - DGAP for IEEE 37 buses

\section{CONCLUSIONS}

A program TS has been obtained that one makes exhaustive selection, analyzing all the possibilities of a configuration in a single iteration. This is important to fortify the mechanism search. Therefore, they are graphical you will climb, instead of the classic geometrically descendent curves.

The implementation of TS for the DGAP throws satisfactory results as far as the time of calculation: 100 iterations in 11/2 minutes or less for six buses, depending of the CPU. And the obtaining of an optimal solution, from the exercise of the fig 6 , without stopping in the optimal premises (Table II). This thanks to the small modification of the main rules of classic TS eliminating the instruction to finalize the process when using an aspiration criteria. The presented model is similar to an exact mathematical model, with the difference of which it uses useful principles like: the punishment of movements made and the search in zones non-explored by means of the diversification.

$\begin{array}{llc}\text { Configuración } & \text { Perfil de Voltajes } & \text { TPL } \\ \text { Bus 1 - CT } & 1.0500+\mathrm{j} 0.0000 & \\ \text { Bus 2 - L } & 1.0208+\mathrm{j} 0.0211 & \\ \text { Bus 3 - L } & 1.0029+\mathrm{j} 0.0541 & \\ \text { Bus 4 - DG } & 1.0500-\mathrm{j} 0.0000 & 0.2087+\mathrm{j} 0.1736 \\ \text { Bus 5 - L } & 0.9717-\mathrm{j} 0.0168 & \\ \text { Bus 6 - DG } & 1.0000+\mathrm{j} 0.0948 & \end{array}$

Table. II. Results after 100 iterations.

Of fig 11 it is possible to be observed that TS-DGAP has optimized of such form that has gotten almost to annul the TPL. It is necessary to give emphasis to the selection system that is implemented in the model. Then thanks to him, with a few iterations reach a practically ideal solution for a SD.

As it's designed to work with the global variables of the system the modification each one of the aspects can be worked of fast form to take into account for the optimization. This allows that the initial data are conserved in the original variables and to be able to observe the evolution of the initial solution (running only the PFA) and the solution found by the modified TS.

\section{REFERENCES:}

[1] Begovic M. M., Radibratovic B., Lambert C. F, “A multiobjetive volt - VAR optimization in power systems", international Proceedings $37^{\circ}$ conference on systems sciences, Hawaii, IEEE, 2004. 
[2] Nara K., Shiose To, Kitagawa M., Ishihara T., "Implementation of genetic algorithm for distribution systems loss minimum re-configuration”, transactions on to power systems, IEEE, volume 7, volume 3, pp 1044 1051, Aug. 1992, INSPEC 4251908.

[3] Nara K., Hayashi And, Ikeda K., Ashizawa T., "Application of tabu search to optimal placement of distributed generators", Power engineering society to winter meeting, $1^{\circ}$ feb 2001, volume 2, pp 918 - 923, INSPEC 7023500.

[4] Mori H., Ogita A., "Parallel tabu search for capacitor placement in radial distribution”, Power engineering society to winter meeting, 23 - 27 Jan 2000, volume 4, pp 2334 - 2339, INSPEC 6670743.

[5] Glover F., Melián B., “Tabu Search”, UPM, www.tornado.diu.fi.upm.es/caepia/numeros/19/glover.pdf

[6] Glover F., Laguna M., “Tabu Search”, www.dei.umpd.it/ fisch/ricop/tabu_search_glover_laguna .pdf

[7] Jayaswal S., "A comparative study of tabu search and simulated annealing travelling salesman problem”, Department of management sciences, University of Waterloo, www.eng.uwaterloo.ca/ sjayaswa/courses.htm.

[8] Alvarado F. L., "Solving power flow problems with a Matlab implementation of the power system application data dictionary”, System sciences, Proceedings of the $32^{\text {nd }}$ annual Hawaii international conference, 5 - 8 Jan 1999, INSPEC 6182129.

[9] Morales E. Búsqueda, optimización y aprendizaje, ccc.inaoep.mx/ emorales/cursos/Busqueda04/principal.ht $\underline{\mathrm{ml}}$

[10] Dugan. R, McDermott T., “Distributed generation”, IEEE industry applications magazine, Mar - Apr 2002.

[11] Lee K.Y., Mohtadi M.A., Ortiz J.L., Park Y.M., “Optimal operation of large - scale power systems", IEEE transactions on power systems, Vol. 3, No. 2, May 1998, INSPEC 3293248.

[12] Radial test feeders - IEEE distribution system analysis subcommittee, www.ewh.ieee.org/soc/pes/dsacom/testfeeders.html2004. 